\title{
Synthesis of liver-targeting dual-ligand modified GCGA/5-FU nanoparticles and their characteristics in vitro and in vivo
}

This article was published in the following Dove Press journal:

International Journal of Nanomedicine

4 November 2013

Number of times this article has been viewed

\author{
Mingrong Cheng ${ }^{1,2, *}$ \\ Xiaoyan $\mathrm{Gao}^{3, *}$ \\ Yong Wang ${ }^{4, *}$ \\ Houxiang Chen ${ }^{5}$ \\ Bing $\mathrm{He}^{6}$ \\ Yingchun $\mathrm{Li}^{2}$ \\ Jiang $\operatorname{Han}^{\prime}$ \\ Zhiping Zhang'
}

'Department of General Surgery, Pudong New Area District Zhoupu Hospital, Shanghai, People's Republic of China; ${ }^{2}$ Department of Endoscopy, ${ }^{3}$ Department of Plastic Surgery, Pudong New Area District Zhoupu Hospital, Shanghai, People's Republic of China; ${ }^{4}$ School of Materials Science and Engineering, Wuhan University of Technology, Wuhan, People's Republic of China; ${ }^{5}$ Zhejiang Huafon Fiber Research Institute, Zhejiang Huafon Spandex Co, Ltd, Wenzhou, People's Republic of China; ${ }^{6}$ Department of General Surgery, Shanghai Fifth People's Hospital, Fudan University, Shanghai, People's Republic of China

*These authors equally contributed to this research

Correspondence: Yong Wang

School of Materials Science and

Engineering, Wuhan University of

Technology,

Wuhan 430070, People's Republic of China

$\mathrm{Tel}+86$ I89 713 74413

Fax +86278 7880734

Email wangyong@whut.edu.cn

Bing $\mathrm{He}$

Department of General Surgery,

Shanghai Fifth People's Hospital,

Fudan University, Shanghai 200240,

People's Republic of China

Tel +86 2124289005

$\mathrm{Fax}+8621643076 \mathrm{II}$

Email 20I Icmr@sina.com

\begin{abstract}
Nanoparticle drug delivery systems using polymers hold promise for clinical applications. We synthesized dual-ligand modified chitosan (GCGA) nanoparticles using lactic acid, glycyrrhetinic acid, and chitosan to target the liver in our previous studies. We then synthesized the GCGA/5-FU nanoparticles by conjugating 5-fluorouracil (5-FU) onto the GCGA nanomaterial, which had a mean particle size of $239.9 \mathrm{~nm}$, a polydispersity index of 0.040 , a zeta potential of $+21.2 \mathrm{mV}$, and a drug loading of $3.90 \%$. GCGA/5-FU nanoparticles had good slow release properties, and the release process could be divided into five phases: small burst release, gentle release, second burst release, steady release, and slow release. Inhibitory effects of GCGA/5-FU on tumor cells targeted the liver, and were time and dose dependent. GCGA nanoparticles significantly prolonged the efficacy of 5-FU on tumor cells, and alleviated the resistance of tumor cells to 5-FU. GCGA/5-FU nanoparticles were mostly concentrated in the liver, indicating that the GCGA nanoparticles were liver targeting. GCGA/5-FU nanoparticles significantly suppressed tumor growth in orthotopic liver transplantation mouse model, and improved mouse survival.
\end{abstract}

Keywords: liver cancer, chemotherapy, targeted therapy, 5-fluorouracil

\section{Introduction}

Cancer is the leading cause of mortality threatening human health and life. Worldwide, liver cancer ranks sixth in terms of incidence, and third in terms of mortality. ${ }^{1}$ In the People's Republic of China, liver cancer is the second most common cancer, with an incidence of 300,000 cases each year, causing about 110,000 deaths, accounting for $45 \%$ of total liver cancer deaths worldwide. ${ }^{2}$ Surgical treatments for early liver cancer include resection and transplantation. However, donor shortages and high expenses often lead to the death of patients prior to a liver donor. Thus, liver cancer is still a major cause of cancer death, and the 5-year survival rate of liver cancer is about $18 \%$. $^{3}$ Twenty-two percent of patients who are unable to undergo liver resection or transplantation often died waiting for treatment. Since the currently available anticancer drugs have a low bioavailability, poor targeting properties, severe toxic effects, and can significantly suppress immune functions. Therefore, finding a drug with high efficiency and low side effects is necessary for treating liver cancer. In recent years, liver-targeting drug delivery systems have become a hotspot in strategies for liver cancer therapy. These systems play an essential role in cancer therapy since they can specifically deliver drugs to liver lesions and not to normal liver tissues, thus allowing decreasing dosage and dose frequency, reducing side effects, and improving treatment outcomes. ${ }^{4,5}$ 
Currently, studies about drug delivery systems mainly focus on single-targeted drug delivery systems, ${ }^{6,7}$ such as the chitosan (CTS)-based drug delivery system, which allows liver-targeting drug delivery using specific interactions between galactose and the asialoglycoprotein receptor (ASGP-R) expressed on liver or hepatoma cells surface. ${ }^{8}$ Another ligand used in liver-targeting drug delivery is glycyrrhetinic acid (GA), which is enriched in liver tissues. Studies detected a large amount of GA binding sites on the surface of rat liver cells, and hepatoma cells significantly expressed more GA receptors than normal liver cells. ${ }^{9}$ Since GA itself can inhibit hepatoma cells proliferation, GA-based livertargeted therapy could effectively suppress tumor growth, and showed satisfactory treatment outcomes. ${ }^{9,10}$ Nevertheless, such single-target modified nanoparticles are affected by a number of physiological or pathological factors during ligand-receptor interactions, causing failure in receptormediated functions and in targeted-therapy outcomes. For example, the density and activity of ASGP-R in patients with liver disease are reduced, thus decreasing the binding efficiency of hepatoma cells by $95 \%,{ }^{11}$ indicating that the effects of liver-targeting therapy mediated by ASGP-R only would significantly decrease or disappear. Also, since not all targeted receptors show specific overexpression in target cells, mono-ligand modified nanoparticles may have insufficient receptor binding. ${ }^{12}$ Hence, single-targeting drug delivery systems may have limited therapeutic effects due to the limited specific recognition and binding between ligand and receptor on the target cells. In a previous study, we successfully synthesized a dual-ligand modified chitosan (GCGA) nanoparticle composed of CTS, GA, and lactobionic acid, and observed that GCGA modified nanoparticles had good liver-targeting properties, which were significantly higher than mono-ligand modified nanoparticles. ${ }^{13}$

The thymidylate synthase inhibitor 5-fluorouracil (5-FU) has been used for half a century as a drug of choice for treating solid tumors. ${ }^{14,15}$ However, this drug requires intravenous injection because of low oral bioavailability and short plasma half-life (only 5 minutes in vivo). In order to reach an effective drug concentration, high doses, and continuous or repeated administration are often used in clinical practice, which in turn increase the toxicity of 5-FU. Also, 5-FU has low selectivity and high toxicity, which may lead to bone marrow suppression, mucositis, diarrhea, hair loss, and liver and kidney damage. ${ }^{16}$ In recent years, many formulations have been tried to improve the selectivity and efficacy of 5-FU, as well as to decrease its toxicity and side effects. Researchers synthesized less toxic 5-FU prodrugs, such as fluorouridine and tegafur, and investigated their pharmacology, toxicity, metabolism, and antitumor activity. Results showed that although these prodrugs had less toxicity and higher chemotherapy index than 5-FU, they still lack tumor selectivity, and can cause adverse effects in nontarget cells and organs. ${ }^{17,18}$ With the use of polymer science in medicine, researchers found that when fixed on polymers, active small-molecule drugs acquired slow release and long-action characteristics, and could effectively regulate the transport and absorption of drugs in vivo. Meanwhile, since these polymer modified drugs can easily be endocytosed by tumor cells, the drugs are target released, and they have significantly fewer toxic side effects. ${ }^{19}$ Recent studies focused on the synthesis of tumor-targeting 5-FU-coated nanoparticles to render targeting properties to 5-FU. We previously synthesized nanoparticles using galactosyl-chitosan (GC) and 5-FU. Results showed that these GC/5-FU nanoparticles had obvious targeting properties against liver cancer, and that they may regulate the distribution and metabolism of 5-FU in vivo, thereby significantly reducing the toxic side effects of 5-FU. ${ }^{20}$ In this study, we successfully synthesized the GCGA/5-FU nanoparticle, and its characteristics were observed in vitro and in vivo. This study used GCGA/5-FU nanoparticles to treat mice with liver cancer, and results showed that GCGA/5-FU nanoparticles significantly inhibited tumor growth and attenuated the immune suppression induced by 5 -FU.

\section{Materials and methods Experimental materials}

Experimental materials used in this study included: CTS ( $>85 \%$ degree of deacetylation, Sigma-Aldrich, St Louis, MO, USA), lactobionic acid ( $\geq 97 \%$ purity, Sigma-Aldrich), GA ( $\geq 97 \%$ purity, Xi'An Fujie Pharmaceutical Co, Ltd, Yilu, People's Republic of China), hydrochloric acid (Shanghai Reagent Company, Shanghai, People's Republic of China), 1-ethyl-3-(3-dimethylaminopropyl) carbodiimide hydrochloride (Sigma-Aldrich), N-hydroxysuccinimide (Sigma-Aldrich), tetramethylethylenediamine (Sinopharm Chemical Reagent Co, Ltd, Shanghai, People's Republic of China), sodium polyphosphate (TPP, $\geq 98 \%$ purity, Aladdin Reagent Co, Ltd, Shanghai, People's Republic of China), and RPMI 1640 powder (Gibco ${ }^{\circledR}$, Life Technologies, Carlsbad, CA, USA).

\section{Experimental animals and cell lines}

Human hepatocellular carcinoma cells (SMMC-7721) and normal liver cells (LO2) were obtained from the 
Committee on Type Culture Collection of the Chinese Academy of Sciences (Shanghai, People's Republic of China). The human colon cancer cell line (SW480) was purchased from the American Type Culture Collection (Manassas, VA, USA). The mouse hepatoma cell line H22 was purchased from the China Center for Type Culture Collection (Wuhan, People's Republic of China). Female $\mathrm{BALB} / \mathrm{c}$ mice, 7 weeks old and weighing about $20 \mathrm{~g}$, were obtained from the Science Department of Experimental Animals of the Fudan University (Shanghai, People's Republic of China). All mice were housed in a specific pathogen-free level B animal facility. Sprague Dawley rats (weighing 60-80 g, aged 21 days) were purchased from the Experimental Animal Center of the Tongji Medical College, Huazhong University (Wuhan, People's Republic of China). The study was approved by the Review Boards of the Shanghai Zhoupu Hospital and the Fudan Medical College.

\section{Preparation of GCGA/5-FU nanoparticles}

The GCGA/5-FU nanoparticles were prepared using an ionic crosslinking technique. 5-FU was added into GCGA solution (dissolved in acetic acid) at a mass ratio of 1:1. An aqueous TPP solution $(0.05 \%[\mathrm{w} / \mathrm{v}])$ was then added to the mixture and stirred. After 50 minutes, GCGA/5-FU nanoparticles were formed by the incorporation of TPP solution at a ratio of GCGA:TPP of 5:1. The nanoparticles were centrifuged at 10,000 rpm for 30 minutes to remove $\mathrm{NaOH}$ and 5-FU. Purified nanoparticles were lyophilized and preserved for future use. The CTS and GA-CTS nanoparticles were prepared in the same way.

\section{Determination of particle size and zeta potential}

The cuvette containing the prepared nanoparticles suspension was placed into the sample chamber for determination of particle size and particle size distribution. A volume of $5 \mathrm{~mL}$ of nanoparticles suspension was injected into the pipeline for measurement of the zeta potential.

\section{Targeting properties of GCGA/5-FU nanoparticles}

We randomly divided Balb/c mice into four groups $(n=5)$, and intravenously injected $200 \mu \mathrm{L}$ of 5 -FU solution (containing $0.371 \mathrm{mg}$ 5-FU), CTS/5-FU, GA-CTS/5-FU or GCGA/5-FU solution in each group. Mice were killed 30 minutes later, and their liver, spleen, kidney, lung, muscle, and heart tissues were sampled, weighed (0.5-1.0 g), and homogenized.
A total of $0.5 \mathrm{~mL}$ of tissue homogenates were taken for detecting 5-FU concentration.

\section{Distribution of GCGA/5-FU nanoparticles}

We randomly divided Sprague Dawley rats into four groups $(n=5)$. Rats in the control group were only injected with saline; rats in the CTS, GA-CTS, and GCGA groups were injected with fluorescein isothiocyanate (FITC)-labeled CTS, GA-CTS or GCGA nanoparticles, respectively. We weighed the rats, injected the drugs, then killed the rats 30 minutes later. Liver, spleen, kidney, lung, muscle, and heart tissues were immediately sampled and frozen in $10 \mu \mathrm{m}$ thick sections. Sections were fixed in 4\% paraformaldehyde, stained with Hoechst 33258 for 5 minutes, washed with phosphate buffered saline (PBS) three times, and observed under confocal microscopy to compare the distribution of green fluorescence between the different groups.

\section{Cytotoxicity of GCGA/5-FU nanoparticles}

SW480 and SMMC-7721 cells were grown in RPMI 1640 medium with $10 \%$ fetal calf serum at $37^{\circ} \mathrm{C}$ in $5 \% \mathrm{CO}_{2}$. Cells $\left(1 \times 10^{4}\right)$ were plated in 96-well plates with $190 \mu \mathrm{L} /$ well and were cultured for 24 hours. Cells were treated with $10 \mu \mathrm{L}$ of 5-FU, CTS/5-FU, GA-CTS/5-FU, or GCGA/5-FU nanoparticles at different doses for 24,48 , or 72 hours. Thirty $\mu \mathrm{g}$ of methyl thiazolyl tetrazolium (MTT, Sigma-Aldrich) was added into each well, and the mixture was incubated for 4 hours. The medium was removed, $200 \mu \mathrm{L}$ of dimethyl sulfoxide (DMSO) was added, and the plate was agitated for 10 minutes. The absorbance was measured using a Bio-Rad automatic microplate reader (Hercules, CA, USA) at $490 \mathrm{~nm}$. All measurements were performed in triplicate. MTT assays were performed at days 4, 5, 6, 7, 8, 9, and 10 for cells treated with $1.6 \mathrm{mg} / \mathrm{L}$ of $5-\mathrm{FU}$. Cytotoxicity was determined by the following equation:

$$
\begin{aligned}
\text { Cytotoxicity }(\%)= & \left(\mathrm{A}_{490} \text { of control }-\mathrm{A}_{490} \text { of sample }\right) / \\
& \mathrm{A}_{490} \text { of control } \times 100 \% .
\end{aligned}
$$

\section{Establishment of SMMC-772I/5-FU drug-resistant cell lines and resistance index calculation}

The drug-resistant cell line SMMC-7721/5-FU was established by the method of concentration gradient increased induction. ${ }^{21}$ Twenty $\mathrm{mL}$ of SMMC-7721 cells at a concentration of $1 \times 10^{4} /$ 
$\mathrm{mL}$ were cultured in the logarithmic phase in an $100 \mathrm{~mL}$ culture flask in RPMI 1640 medium with 10\% neonatal bovine serum (NBS) at $37^{\circ} \mathrm{C}$ in $5 \% \mathrm{CO}_{2}$ and cultured for 24 hours. The cultured solution was replaced with 5 -FU at a concentration of $3.2 \mathrm{mg} / \mathrm{mL}$. After 48 hours of culture, the solution containing drugs was discarded and replaced with 5-FU solution at a lower concentration $(0.8 \mathrm{mg} / \mathrm{mL})$; the process was then repeated three times. Cells were alternatively treated with 5-FU solutions at different concentrations (6.4, 1.6, and $12.8 \mathrm{mg} / \mathrm{mL}$ ). After 2 months, cells in the logarithmic phase were cultured in the medium with $3.2 \mathrm{mg} / \mathrm{mL}$ of 5 -FU.

Two hundred $\mu \mathrm{L}$ of SMMC-7721/5-FU drug-resistant cells and normal SMMC-7721 cells were plated into 96-well plates at a concentration of $1 \times 10^{4} / \mathrm{mL}$. After incubation for 24 hours, cells were centrifuged and collected. SMMC-7721/5-FU drug-resistant cells were reinoculated in different doses of 5-FU $(0,3.2,6.4,12.8,25.6,51.2$, and $102.4 \mathrm{mg} / \mathrm{L}, \mathrm{n}=7)$. SMMC-7721 cells were incubated with lower concentrations of $5-\mathrm{FU}(0,0.2,0.4,0.8,1.6,3.2$, and $6.4 \mathrm{mg} / \mathrm{L}, \mathrm{n}=7$ ). After 48 hours, the absorbance was measured at $490 \mathrm{~nm}$ by the MTT method. The half maximal inhibitory concentration $\left(\mathrm{IC}_{50}\right)$ values and cytotoxicity were determined. Resistance index (RI) was determined as:

$$
\begin{gathered}
\mathrm{RI}=\mathrm{IC}_{50} \text { value of drug-resistant cell/ } \\
\quad \mathrm{IC}_{50} \text { value of parental cells. }
\end{gathered}
$$

\section{Inhibition of GA-CTS/5-FU nanoparticles on SMMC-772I/5-FU drug-resistant cells}

Two hundred $\mu \mathrm{L}$ of SMMC-7721/5-FU cells were plated into 96-well plates at a concentration of $1 \times 10^{4} / \mathrm{mL}$. After incubation for 24 hours, cells were centrifuged and collected. Cells were treated with 5-FU $(3.2,6.4,12.8,16,19.2,22.4$, and $25.6 \mathrm{mg} / \mathrm{L}$ ) and GA-CTS/5-FU nanoparticles were added at the same doses and incubated for 48 hours. In the other two groups, cells were treated with $6.4 \mathrm{mg} / \mathrm{mL}$ of $5-\mathrm{FU}$ and GA-CTS/5-FU nanoparticles, respectively. Cells were cultured for 12, 24, 36, 48, 60, and 72 hours. MTT assays were performed to determine the cytotoxicity of the hepatocellular carcinoma cells.

\section{Inhibitory effects of GCGA/5-FU nanoparticles in orthotopic liver transplantation mouse model}

Five days after establishment of the orthotopic liver transplantation mouse model, tumor size reached $4-6 \mathrm{~mm}$. Six days after model establishment, mice were divided into six groups: control, GCGA, 5-FU, CTS/5-FU, GA-CTS/5-FU and GCGA/5-FU. Mice in the six groups were injected with saline, GCGA, 5-FU CTS/5-FU, GA-CTS/5-FU, or GCGA/5-FU nanoparticles, respectively, at a volume of $200 \mu \mathrm{L}$ (all 5-FU-containing injections contained $0.371 \mathrm{mg}$ of 5 -FU). Drugs were administered for 5 days, and 10 mice in each group were killed at 10 days after the first dose to measure tumor growth and tumor weight. The remaining 13 mice in each group were used for survival analysis.

\section{Data analysis}

All data are expressed as mean \pm standard deviation (SD). One-way analysis of variance and the least significant difference test were used for comparisons between groups. Kaplan-Meier survival plots were used for survival analysis. A $P$-value $<0.05$ was considered significant.

\section{Results}

\section{Sustained release property of the GCGA/5-FU nanoparticles}

We synthesized successfully the liver/tumor-targeting GCGA/5-FU nanoparticles (Figure 1A), which had a mean particle size of $239.9 \mathrm{~nm}$, a polydispersity index of 0.040 , a zeta potential of $+21.2 \mathrm{mV}$ (Figure 1B), and a drug loading of $3.90 \%$.

We conducted in vitro experiments to investigate the 5-FU releasing characteristics of the GCGA/5-FU nanoparticles. The drug-release curve indicated that when 5-FU concentration was between 0.1 and $20 \mathrm{mg} / \mathrm{L}$, it had a good linear relationship with the 5-FU peak, and the regression equation was:

$$
\mathrm{y}=2.5721 \mathrm{x}+0.6851(r=0.9956)
$$

Figure $1 \mathrm{C}$ shows that the 1-hour cumulative release rate of 5-FU reached $95.67 \%$ in vitro. The release of GCGA/5-FU could be divided into five phases: 1 ) the small burst release phase ( $0-20$ minutes), with a cumulative release rate of $4.9 \%$ at 20 minutes; 2 ) the gentle release phase (20-220 minutes), with a cumulative release rate of $4.9 \%-7.2 \%$ (2.3\% increment in cumulative release rate); 3 ) the second burst release phase (220-280 minutes), with a cumulative release rate of $7.2 \%-28.5 \%$ (21.3\% increment in cumulative release rate); 4) the steady release phase ( $0.2-6$ days), with a cumulative release rate of $28.5 \%-89.1 \%(60.6 \%$ increment in cumulative release rate); and 5) the slow release phase (6-10 days), with a cumulative release rate of $89.1 \%-96.8 \%$ (7.7\% increment in cumulative release rate). 


\section{A}

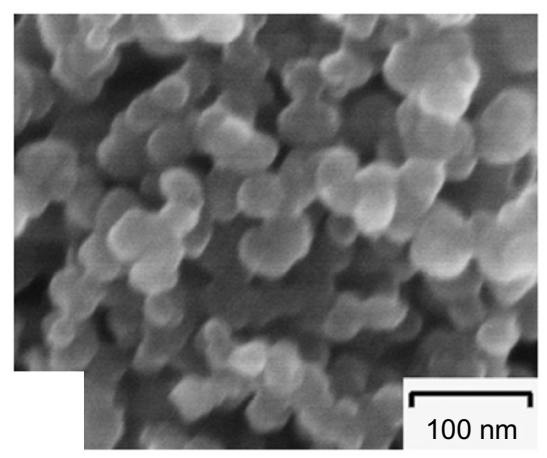

B

z-average size $(\mathrm{nm}): 239.9$ Poly index: 0.04

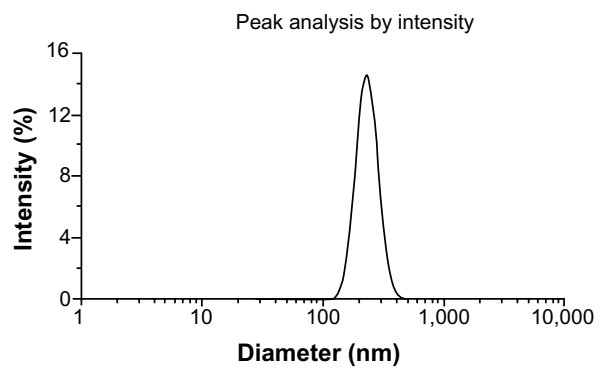

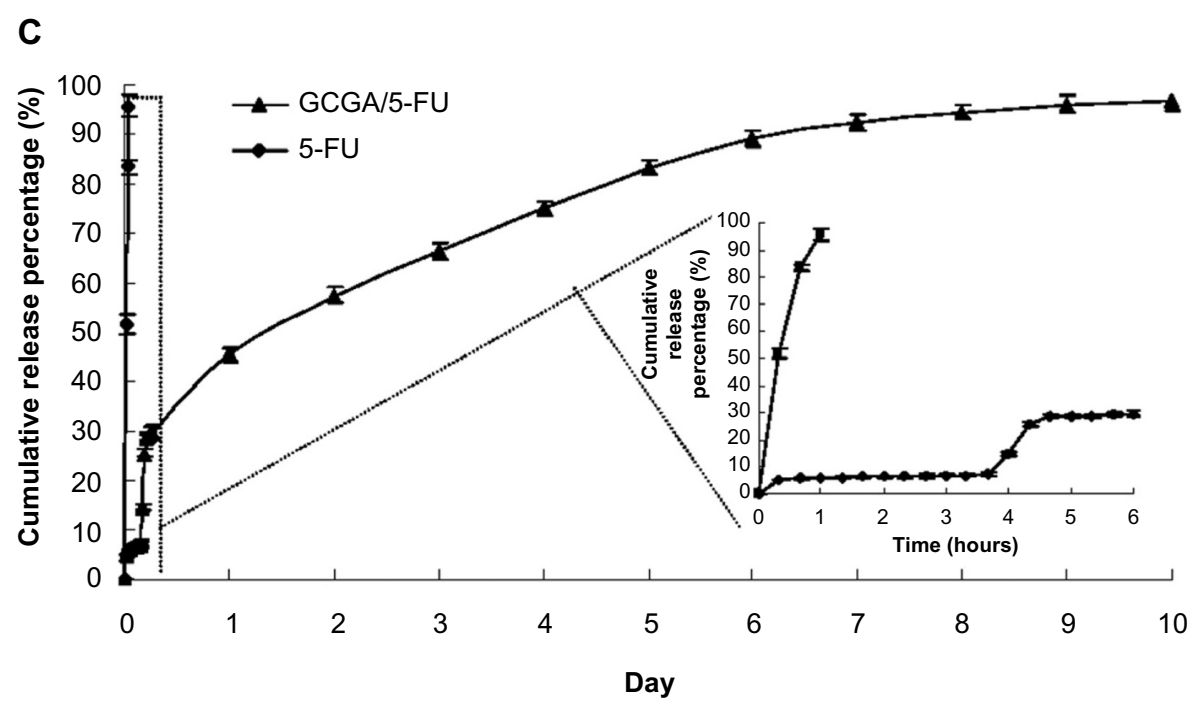

D
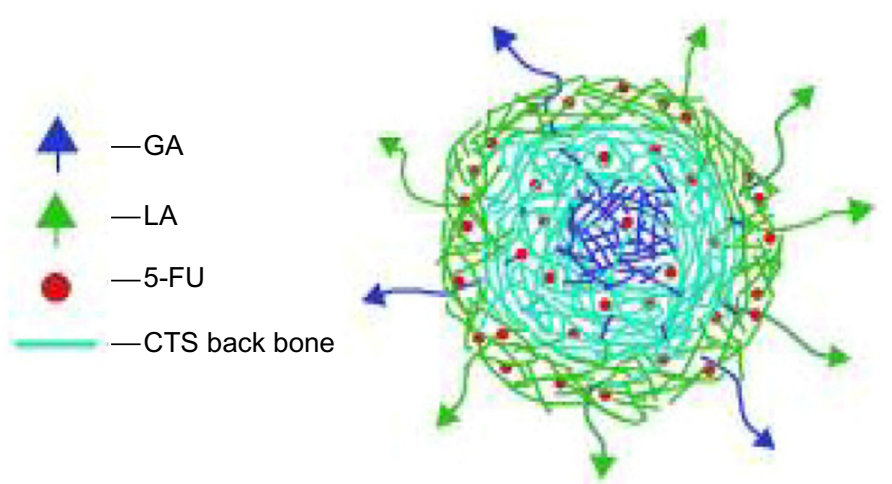

Figure I Characteristics of GCGA/5-FU nanoparticles.

Notes: (A) Electron micrograph of GCGA/5-FU nanoparticles. (B) Particle size of GCGA/5-FU nanoparticles. (C) In vitro releasing curve of GCGA/5-FU nanoparticles in simulated body fluid $\left(37^{\circ} \mathrm{C}, \mathrm{pH}=7.4\right)$. Data are presented as mean $\pm \mathrm{SD}(\mathrm{n}=3)$. (D) Hypothetical figure of $\mathrm{GCGA} / 5-\mathrm{FU}$ nanoparticles.

Abbreviations: 5-FU, 5-fluorouracil; CTS, chitosan; GA, glycyrrhetinic acid; GCGA, dual-ligand modified chitosan; h, hours; LA, lactic acid; SD, standard deviation.

\section{In vivo distribution of GCGA/5-FU nanoparticles}

Previous in vitro studies confirmed that GCGA could target both liver and hepatoma cells, and our study aimed to investigate whether GCGA can redistribute drugs in vivo. Figure $2 \mathrm{~A}$ shows that the liver had the highest 5-FU concentration among investigated tissues, and that the GCGA/5-FU nanoparticles were most concentrated in the liver, followed by GA-CTS/5-FU, CTS/5-FU, and 5-FU. The liver concentration of 5-FU in the GCGA/5-FU group was 1.65, 2.35, and 2.75 times of the GA-CTS/5-FU, CTS/5-FU, and 5-FU groups, respectively. However, 5-FU concentration in lung, kidney, heart, brain, and muscle tissues exhibited a reversed trend, with the 5-FU groups having the highest 5-FU concentrations, followed by the CTS/5-FU, GA-CTS/5-FU, and GCGA/5-FU groups. 
A
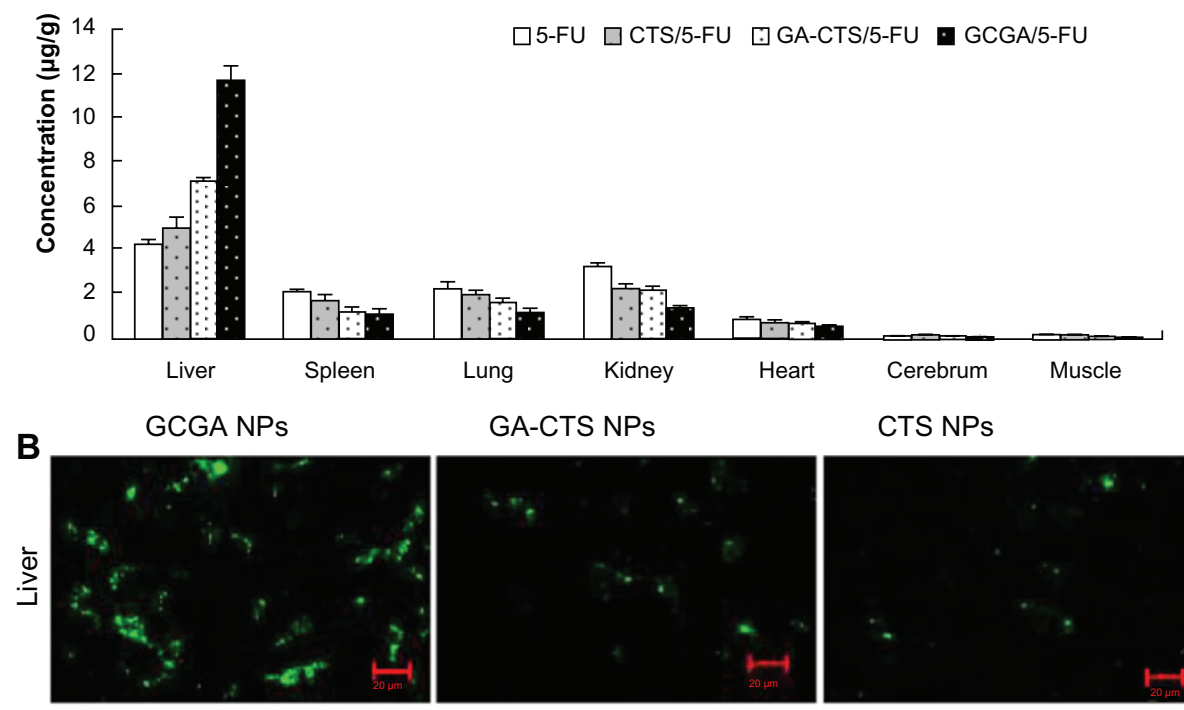

GA-CTS NPS

CTS NPS
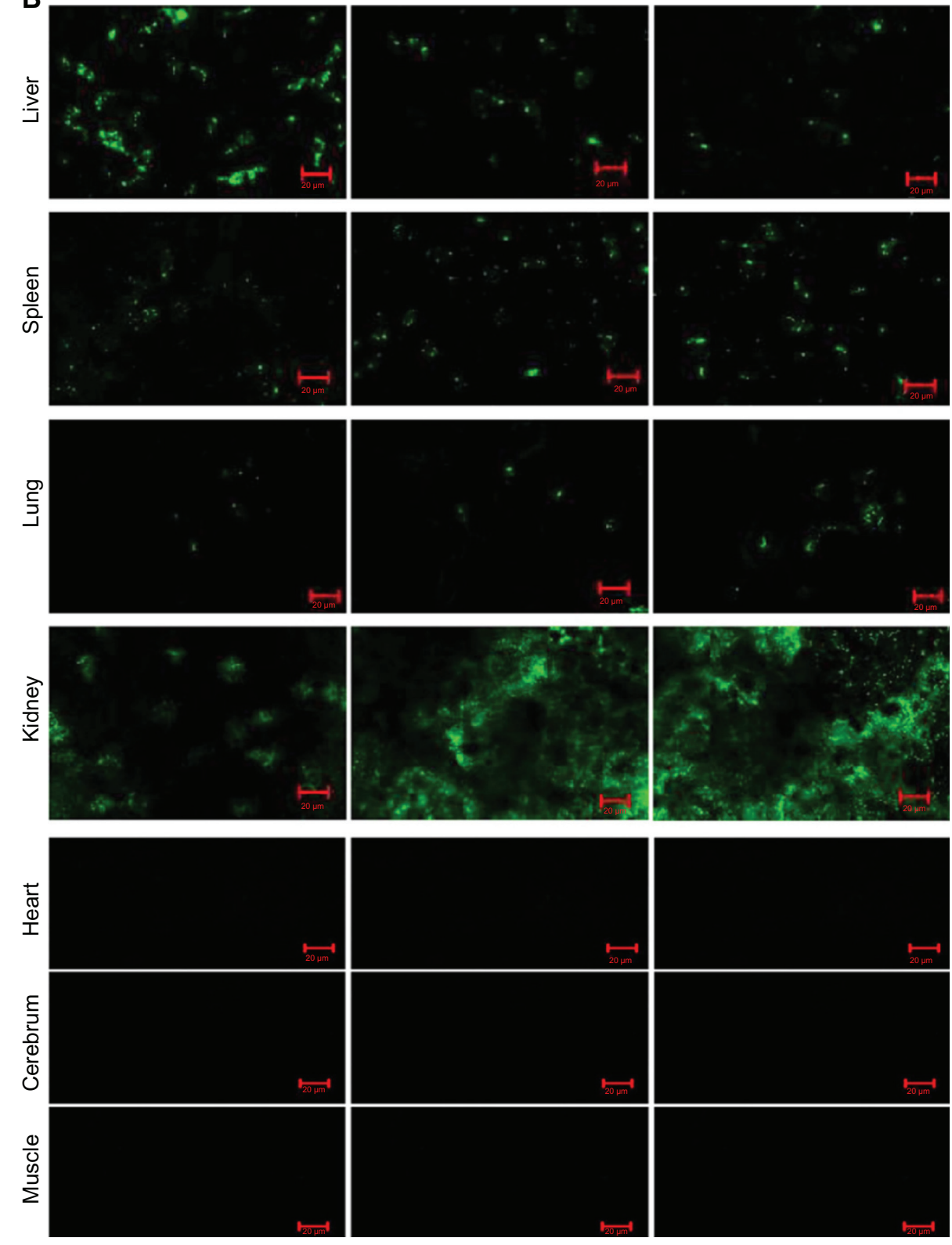

Figure 2 Targeting distribution of GCGA nanoparticles.

Notes: (A) Balb/c mice were assigned to four groups and were injected with 5-FU, GA-CTS/5-FU, CTS/5-FU, or GCGA/5-FU. Mice were killed after 30 minutes for detection of 5-FU concentrations. Data are presented as mean \pm SD $(n=5)$. (B) SD mice were assigned to four groups and were injected with FITC-labeled CTS, GA-CTS, or GCGA nanoparticles. Mice were killed after 30 minutes, and their liver, spleen, lung, kidney, heart, brain, and muscle tissues were taken for confocal microscopy ( $\mathrm{n}=5$ ).

Abbreviations: 5-FU, 5-fluorouracil; CTS, chitosan; FITC, fluorescein isothiocyanate; GA, glycyrrhetinic acid; GCGA, dual-ligand modified chitosan; NP, nanoparticle; $\mathrm{SD}$, standard deviation. 
Confocal microscopy demonstrated that all three groups showed green fluorescence in the kidneys; the CTS nanoparticles had the highest fluorescence signal, followed by the mono-ligand modified GC-CTS nanoparticles and the dualligand modified GCGA nanoparticles (Figure 2B). This trend was also observed in spleen, lung, heart, brain, and muscle tissues. However, a reverse trend was observed in the liver, with the GCGA group having the highest fluorescence signal, followed by the GA-CTS and CTS groups.

\section{Inhibitory effects of GCGA/5-FU nanoparticles in tumor cells are dose and time dependent, and specifically target hepatoma cells}

Figure $3 \mathrm{~A}-\mathrm{C}$ shows that the inhibitory rates in SW480 and SMMC-7721 cells were significantly elevated with increases in 5-FU concentrations. Inhibitory rates of different nanoparticles were detected at 24, 48, and 72 hours, and the results indicated that the hepatic tumor-targeted nanoparticles had the highest inhibitory rate in SMMC7721 cells (GCGA/5-FU-SMMC-7721 > GA-CTS/5FU-SMMC-7721), followed by 5-FU in SW480 and SMMC-7221 cells. The lowest inhibitory rate occurred in the hepatic tumor-targeting GCGA/5-FU and GA-CTS/5FU nanoparticles in SW480 cells, and in the nonhepatic tumor-targeting CTS/5-FU nanoparticles in SW480 and SMMC-7221 cells. The GCGA/5-FU nanoparticles had the highest inhibitory rate in SMMC-7221 cells, followed by the GA-CTS/5-FU nanoparticles. These results suggested that the GCGA/5-FA nanoparticles had significant hepatic tumor-targeting properties; dual-ligand modified nanoparticles had higher inhibitory effects on tumor cells than mono-ligand modified nanoparticles. Nontargeted nanoparticles had a significantly reduced inhibitory effect on tumors compared with 5-FU.

Figure 3D shows that the GCGA/5-FU nanoparticles had the highest inhibitory rate in SMMC-7721 cells, followed by the GA-CTS/5-FU nanoparticles. At 1-5 days, 5-FU had a significantly higher inhibitory rate in SW480 and SMMC7721 cells than GCGA/5-FU (in SW480), GA-CTS/5-FU (in SW480), CTS/5-FU (in SW480), and CTS/5-FU (in SMMC-7721). However, at 6-10 days, the inhibitory rate of 5-FU showed a plateau phase, while nanoparticles showed drastically increased inhibitory rates, which were significantly higher than 5-FU, due to the slow release of the drug. Results suggested that 5-FU from nontargeting nanoparticles could be released slowly, and would not show a plateau phase, indicating that the nontargeting nanoparticles could significantly prolong the efficacy of 5-FU.

\section{GCGA/5-FU nanoparticles enhanced the toxicity in drug-resistant hepatoma cells}

In order to study the toxicity of GCGA/5-FU nanoparticles in drug-resistant hepatoma cells, we successfully established a hepatoma cell line with moderate drug resistance. We used 5-FU to test the resistance of normal and resistant SMMC7221 cells, and the $\mathrm{IC}_{50}$ for the two cell lines was $6.76 \mathrm{mg} / \mathrm{L}$ and $32.34 \mathrm{mg} / \mathrm{L}$, respectively. The RI was calculated as 8.60, indicating that the resistant SMMC-7221 cell line was moderately drug-resistant (Table 1 ).

Figure 3E shows that the inhibition of resistant hepatoma cells increased with drug concentration. Under the same drug concentration, GCGA/5-FU had the highest tumor inhibitory rate, followed by GA-CTS/5-FU and 5-FU (all $P<0.01$ ). Figure $3 \mathrm{~F}$ shows that under the same drug concentration, the inhibitory rate of each group increased with time. At the same time point, GCGA/5-FU nanoparticles had the highest inhibitory rate, followed by GA-CTS/5-FU and 5-FU (all $P<0.01$ ). These results suggested that GCGA/5-FU nanoparticles exhibited an anti-drug resistance potential, since it had a strong inhibitory effect in drug-resistant hepatoma cells.

\section{GCGA/5-FU nanoparticles inhibited mice live tumor growth and improved mice survival}

Ten days after drug injection, mice were killed for tumor tissue sampling and weighing (Figure 4A). The mice in the GCGA/5-FU group had the lowest tumor weight, followed by the GA-CTS/5-FU, CTS/5-FU, and 5-FU groups, and the control had the highest tumor weight (all $P<0.05$ ). There were no significant differences between the control and GCGA groups $(P>0.05)$. Figure 4B shows that the highest cell density, cell proliferation, and division are obvious in control group and GCGA group, and there is the decreasing tendency from the 5-FU, CTS/5-FU, GA-CTS/5-FU, and GCGA/5-FU groups in turn; the tumor cell necrosis and fibrosis demonstrated opposite trends, and the control and GCGA group presented less necrosis and fibrosis, while there was obvious fibrosis in the GCGA/5-FU group.

After orthotopic liver transplantation, mice were randomly assigned to four groups $(\mathrm{n}=13)$, and were treated as described before. Survival analysis was performed using Kaplan-Meier curves (Figure 4C). Mice in the control group started to die 11 days after model establishment, and were all dead on day 20 , showing a median survival time of 16 days. Mice in the 

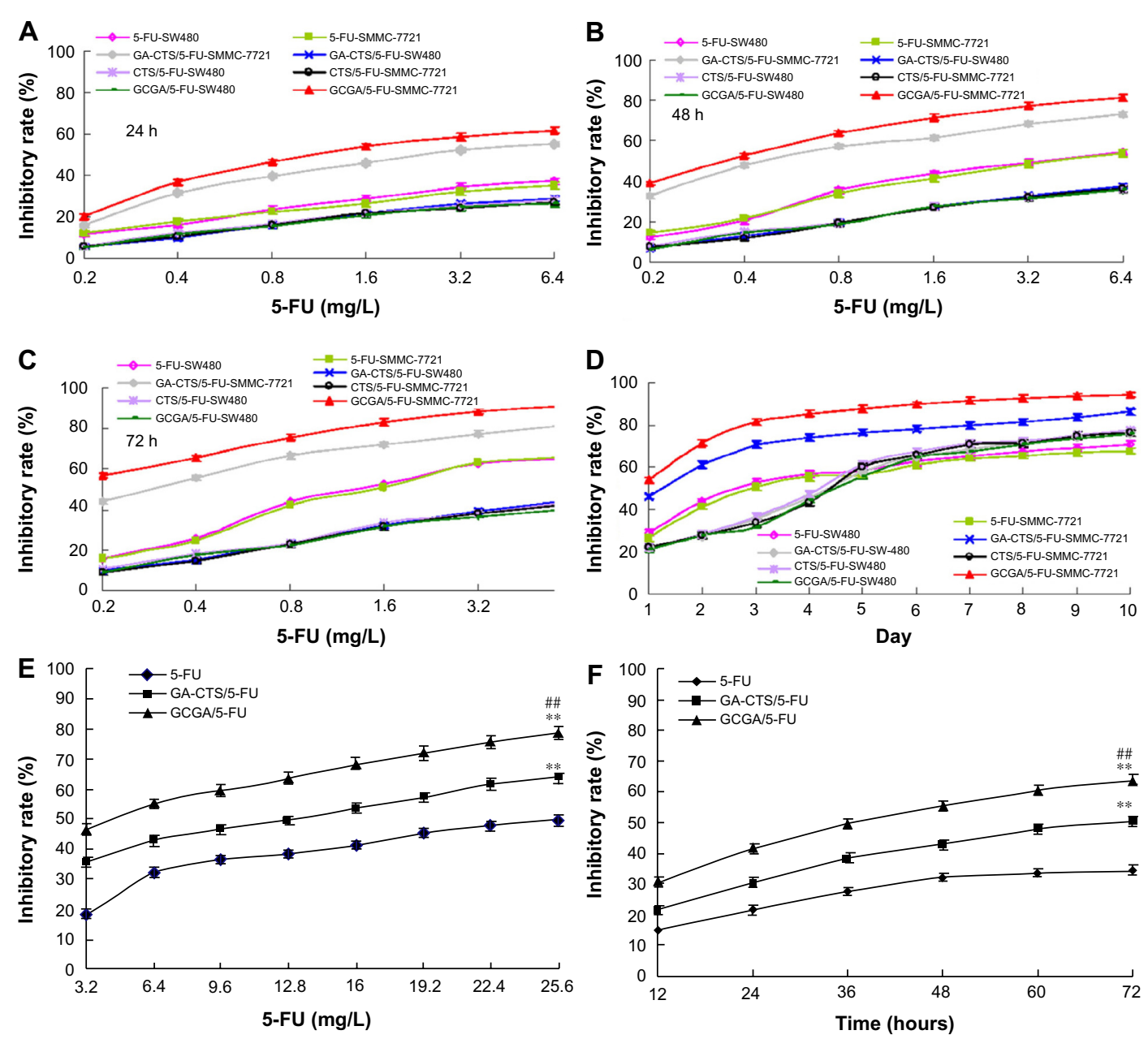

Figure 3 Measurements of inhibitory rate of GCGA/5-FU nanoparticles in SW480 and SMMC-772I cells (non- or 5-FU-resistant) using MTT assays. Data are presented as mean \pm SD $(n=3)$.

Notes: (A) Inhibitory rate curve of different concentrations of 5-FU in SW480 and SMMC-772I cells at 24 hours. (B) Inhibitory rate curve of different concentrations of 5-FU in SW480 and SMMC-772I cells at 48 hours. (C) Inhibitory rate curve of different concentrations of 5-FU in SW480 and SMMC-772I cells at 72 hours. (D) Inhibition rates of different groups on SW480 and SMMC-772I cells after I to 10 days (5-FU: I.6 mg/L). (E) Inhibitory rate curve of different concentrations of 5-FU in 5-FU-resistant SMMC-772I cells. (F) Inhibitory rate curve of 5 -FU $(6.4 \mathrm{mg} / \mathrm{mL})$ in 5 -FU-resistant SMMC-772I cells at different time points. $* * P<0.01$ compared with the 5 -FU group; $\# P<0.01$ compared with the GA-CTS/5-FU group.

Abbreviations: 5-FU, 5-fluorouracil; CTS, chitosan; GA, glycyrrhetinic acid; GCGA, dual-ligand modified chitosan; h, hours; MTT, tetrazolium dye; SD, standard deviation; SMMC-772I, human hepatocellular carcinoma cells.

GCGA group started to die 11 days after model establishment, and were all dead on day 21 , showing a median survival time of 17 days. Mice in the 5-FU group started to die 18 days after model establishment, and were all dead on day 30, showing a median survival time of 24 days. Mice in the CTS/5-FU group started to die 20 days after model establishment, and were all dead on day 37 , showing a median survival time of 30 days. Mice in the GA-CTS/5-FU group started to die 22 days after model establishment, and were all dead on day 44, showing a median survival time of 37 days. Finally, mice in the GCGA/5FU group started to die 25 days after model establishment, and were all dead on day 58 , showing a median survival time of 45 days. The survival time of mice in the GCGA/5-FU group was the longest $(P<0.01)$. These results suggested that the therapeutic effects of liver-targeting dual-ligand modified nanoparticles significantly surpassed the efficacy of monoligand modified nanoparticles. However, the mono modified nanoparticles were still better than nonliver-targeting nanoparticles. Results indicated that nanoparticles could improve treatment outcomes and prolong survival time, and that the liver-targeting dual modified nanoparticles could most significantly improve drug efficacy and prolong survival.

\section{Discussion}

Nanoparticle size and drug loading are two important parameters for a drug delivery system. The pore size of normal vascular endothelial cells is about $2 \mathrm{~nm}$, and the pore size of postcapillary venules is about $6 \mathrm{~nm}$. Liver endothelial cells have pore sizes ranging from 106 to $175 \mathrm{~nm}$, while hepatoma cells have pore sizes from 380 to $580 \mathrm{~nm} \cdot{ }^{22-24}$ An 
Table I Inhibitory effects of 5-FU on SMMC-772I proliferation of parental cell line and 5-FU resistance cell line (mean $\pm \mathrm{SD}, \mathrm{n}=3$ )

\begin{tabular}{lccc}
\hline $\begin{array}{l}\text { Concentrations } \\
\text { (mg/L) }\end{array}$ & A value & $\begin{array}{l}\text { Inhibitory } \\
\text { rate }(\%)\end{array}$ & $\begin{array}{l}I_{50} \\
(\mathbf{m g} / \mathbf{L})\end{array}$ \\
\hline $\begin{array}{l}\text { Parental cell line of SMMC-772 I } \\
0\end{array}$ & & \\
0.2 & $0.65 \pm 0.06$ & & 3.76 \\
0.4 & $0.55 \pm 0.05$ & 14.64 & \\
0.8 & $0.51 \pm 0.07$ & 21.65 & \\
I.6 & $0.43 \pm 0.04$ & 33.65 & \\
3.2 & $0.38 \pm 0.03$ & 41.25 & \\
6.4 & $0.33 \pm 0.03$ & 48.65 & 32.34 \\
5 -FU resistance of SMMC-772I cell line & \\
0 & $0.30 \pm 0.04$ & 53.64 & \\
3.2 & $0.83 \pm 0.06$ & & \\
6.4 & $0.67 \pm 0.05$ & 18.34 & \\
I2.8 & $0.56 \pm 0.06$ & 32.1 & \\
25.6 & $0.51 \pm 0.05$ & 38.24 & \\
51.2 & $0.42 \pm 0.04$ & 49.6 & \\
I02.4 & $0.39 \pm 0.04$ & 53.2 & \\
\hline
\end{tabular}

Abbreviations: 5-FU, 5-fluorouracil; A value, absorbance value; $\mathrm{IC}_{50}$, half maximal inhibitory concentration; SD, standard deviation; SMMC-772I, human hepatocellular carcinoma cells.

ideal nanoparticle size should be larger than the largest pore size of normal liver cells and smaller than the smallest pore size of hepatoma cells. Thus, the nanoparticle size should be between 175 and $380 \mathrm{~nm}$. Drug loading is another important parameter for a drug delivery system. The more drugs a nanoparticle can carry, the higher drug loading it will possess. Thus, many studies have been done to find nanoparticles with high drug loading through rational experimental design and optimization. In this study, GCGA/5-FU nanoparticles had a mean particle size of $239.9 \mathrm{~nm}$, a polydispersity index of 0.040 , a zeta potential of $+21.2 \mathrm{mV}$ (Figure 1B), and a drug loading of $3.90 \%$, and we obtained optimized nanoparticles with relatively small particle size and high drug loading.

We used simulated body fluid to investigate the releasing pattern of GCGA/5-FU nanoparticles. Figure $1 \mathrm{C}$ shows that in vitro release of GCGA/5-FU could be divided into five phases. 1) The small burst release phase (0-20 minutes, with a cumulative release rate of $4.9 \%$ at 20 minutes) can be explained by residual 5-FU during nanoparticle preparation. 2) The gentle release phase (20-220 minutes, with a cumulative release rate of $4.9 \%-7.2 \%, 2.3 \%$ increment in cumulative release rate), which brings the gentle release of drug mainly through diffusion from the nanoparticles. 3) The second burst release phase (220-280 minutes, with a cumulative release rate of $7.2 \%-28.5 \%, 21.3 \%$ increment in cumulative release rate) comes after the gentle release phase. Figure 1D shows that the GCGA/5-FU nanoparticles had a 3-layer structure, with the inner layer composed of hydrophobic GA and insoluble CTS derivatives that could only attract a small amount of hydrophilic 5-FU. However, the outer layer is composed of hydrophilic lactobionate and CTS branches that can coat the drug and retain it in the matrix. Thus, the burst of drug in the third phase may be due to the degradation of the particle outer layer, leading to the breaking of hydrogen bonds between 5-FU and polymers, and the drugs retained in the outer layer are then rapidly dissociated and released. 4) The steady release phase (0.2-6 days, with a cumulative release rate of $28.5 \%-89.1 \%, 60.6 \%$ increment in cumulative release rate) that follows the second burst release phase could be explained by the continuous release of drugs from the degraded insoluble or hydrophobic materials. 5) The final stage, the slow release phase (6-10 days, with a cumulative release rate of $89.1 \%-96.8 \%, 7.7 \%$ increment in cumulative release rate), could be attributed to the slow release of 5-FU from the residual nanoparticles. Our results suggested that the GCGA/5-FU showed significant slow release properties.

Previous studies confirmed that GCGA nanoparticles had in vitro liver-targeting properties. ${ }^{20}$ Figure $2 \mathrm{~A}$ shows that GCGA/5-FU nanoparticles had the highest liver concentration, which was $1.65,2.35$, and 2.75 times the concentrations of the GA-CTS/5-FU, CTS/5-FU, and 5-FU nanoparticles, respectively. However, the concentration of 5-FU in lung, spleen, heart, brain, and muscle tissues decreased from the 5-FU, CTS/5-FU, and GA-CTS/5-FU groups to the GCGA/5-FU group, and this trend was consistent with the results from confocal microscopy, as shown in Figure 2B. The results can be explained, as the dual-ligand modified GCGA/5-FU nanoparticles had higher liver targeting properties than the mono-ligand modified GA-CTS and nonligand modified CTS nanoparticles. In addition, results suggested that ligand modified GA-CTS and GCGA particles both had good liver-targeting properties, but that GCGA particles had higher liver specificity. This was because under pathological conditions, the galactose receptor binding activity would decline, ${ }^{11}$ and the GA receptor had higher expression in cancer cells. ${ }^{9}$ Since the GCGA nanoparticles are modified with the ligands of both target receptors, they possessed a stronger liver-targeting property compared with other monoligand modified nanoparticles.

In terms of tumor-targeting properties of the GCGA/5FU nanoparticles, our study found that with increases in 5-FU concentrations, the inhibitory effect on SW480 and SMMC-7721 also significantly increased. At 24,48 , and 72 hours, the liver-targeting nanoparticles had the highest 


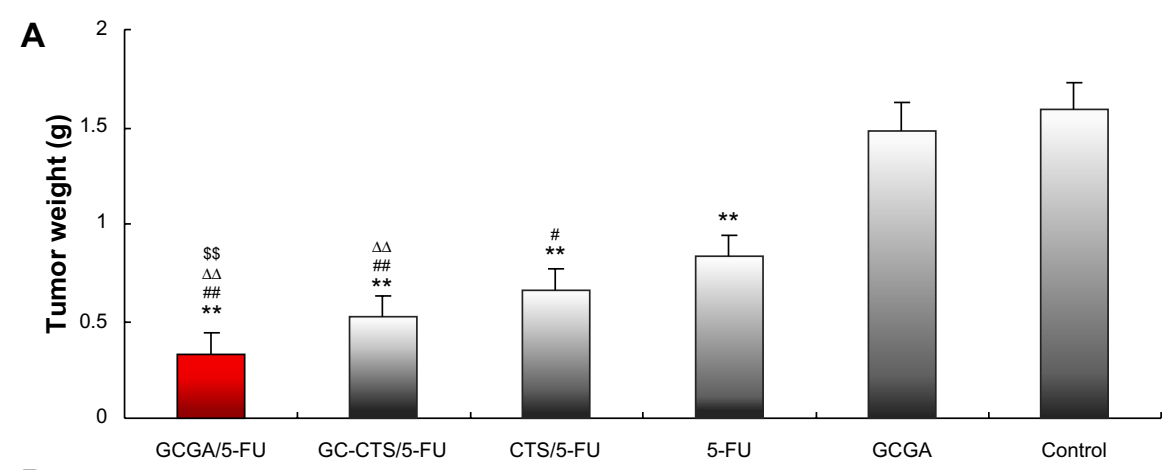

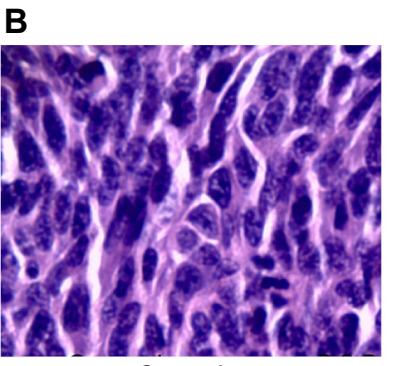

Control

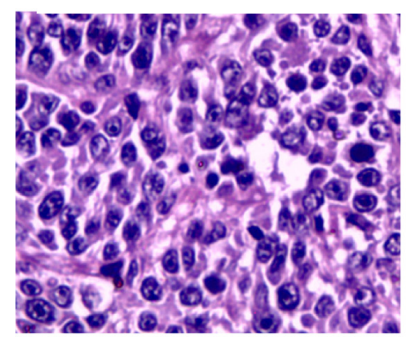

CTS/5-FU

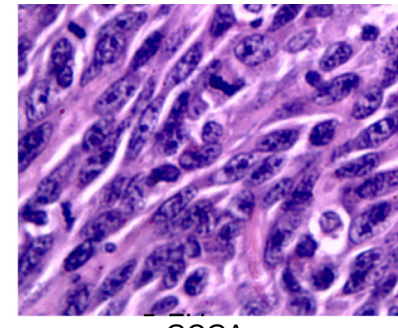

GCGA

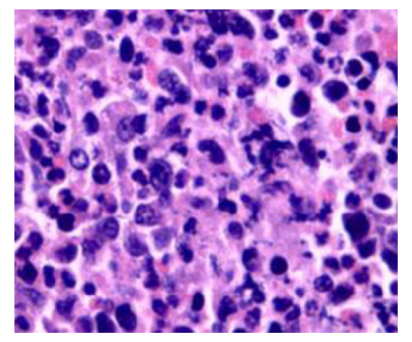

GA-CTS/5-FU

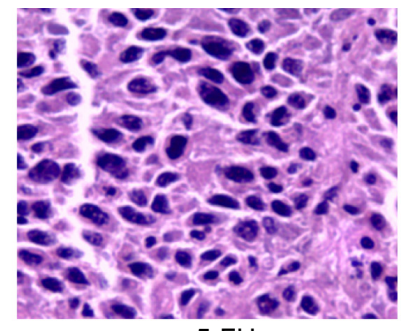

$5-\mathrm{FU}$

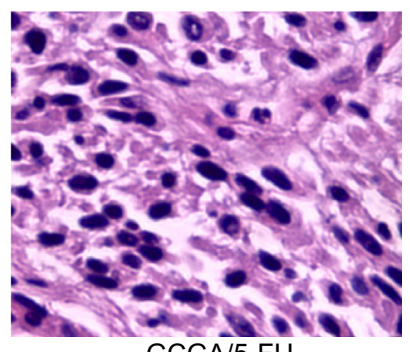

GCGA/5-FU

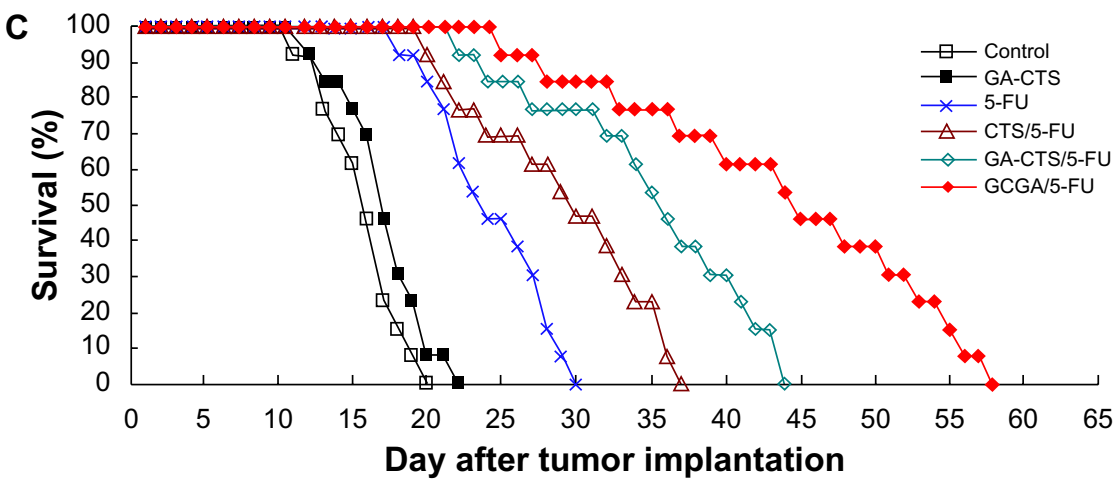

Figure 4 Inhibition by GCGA/5-FU nanoparticles in orthotopic liver transplantation mouse model.

Notes: (A) At 5 days after model establishment, mice were treated with GCGA/5-FU, GA-CTS/5-FU, CTS/5-FU, 5-FU, GCGA, or PBS. Mice were killed 10 days after treatment, and tumor tissues were sampled and weighed. Data are presented as mean $\pm S D(n=10)$. (B) Pathological section of HE stain in different groups $(\times 400)$. (C) After model establishment, mice in each group were treated as described, and Kaplan-Meier curves were used to analyze mice survival. Mice in the GCGA/5-FU group had the longest survival, followed by the GA-CTS/5-FU, CTS/5-FU, 5-FU, and control groups. ${ }^{* * P}<0.01$ compared with control; ${ }^{*} P<0.05$ and ${ }^{\# P} P 0.01$ compared with the 5 -FU group; $\triangle \triangle P<0.01$ compared with the CTS/5-FU group; $\$ \$ P<0.01$ compared with the GA-CTS/5-FU group.

Abbreviations: 5-FU, 5-fluorouracil; CTS, chitosan; GA, glycyrrhetinic acid; GCGA, dual-ligand modified chitosan; h, hours; HE, hematoxylin and eosin stain; PBS, phosphate buffered saline; SD, standard deviation.

inhibitory rate in SMMC-7721 cells, and the inhibitory rate of GCGA/5-FU exceeded the effect of the GA-CTS/5-FU nanoparticles, indicating that when compared with mono modified GA-CTS nanoparticles, the dual modified nanoparticles induced stable endocytosis of 5-FU, resulting in a higher concentration of 5-FU and a higher tumor cell inhibitory rate.
The liver-targeting (GCGA/5-FU and GA-CTS/5-FU) nanoparticles and the nonliver-targeting (CTS/5-FU) nanoparticles had the lowest inhibitory rate in SW480 cells and in SW480/ SMMC-7721 cells, respectively. Although GCGA and GACTS nanomaterial targeted the liver, the SW480 cells were not targeted and the nanoparticles could not induce drug 
endocytosis, resulting in nontargeted therapy by the CTS/5FU nanoparticles in SW480 and SMMC-7721 cells. Also, due to the slow release of 5-FU, the concentration of 5-FU remained low in the tumor cells, and explained the low inhibitory rate of the GCGA and GA-CTS nanoparticles in tumor cells. Figure 3D showed that at $1-5$ days, GCGA/5-FU and GA-CTS/5-FU nanoparticles had the highest inhibitory rate in SMMC-7721 tumor cells, and that the inhibitory effect of 5-FU in SW480 and SMMC-7721 cells was significantly higher than GCGA/5-FU in SW480, GA-CTS/5-FU in SW480, CTS/5-FU in SW480, and CTS/5-FU in SMMC7721 cells. At 6-10 days, the inhibitory rate of the GCGA/5FU and GA-CTS nanoparticles in SMMC-721 cells still remained the highest, while the inhibitory rate of 5-FU in SW480 and SMMC-7721 cells showed a plateau phase, which may due to the short effective time of 5-FU. However, since 5-FU can be slowly released from the GCGA/5-FU, GACTS/5-FU, and CTS/5-FU nanoparticles, the inhibitory rates of these nanoparticles gradually increased instead of showing a plateau phase. Thus, the nanoparticles could significantly prolong the efficacy of 5-FU in tumor cells.

We established a 5-FU resistant SMMC-7721 cell line to investigate the anti-drug resistance potential of the GCGA/5FU nanoparticles. Generally, cell lines with low, moderate, and high resistance show a drug $\mathrm{RI}<5$, between 5 and 15, and $>15$, respectively. ${ }^{25}$ The 5-FU resistant SMMC-7721 cell line established in this study was a moderate resistant cell line, since it had a RI of 8.60. Our study found that GCGA/5-FU could significantly inhibit the proliferation of 5-FU resistant SMMC7721 cells in vitro, and such inhibition was obviously more effective than when using 5-FU alone. These results indicated that the SMMC-7721 cells are resistant to free 5-FU. The metabolite of 5-FU, 5-fluorouracil oligodeoxynucleotide, inhibits thymidylate synthase synthesis, thus preventing the methylation of dUMP to dTMP, blocking DNA synthesis. Water and lipid solubility of 5-FU are very low due to its unique structure, and resistance to 5-FU can be attributed to cell membrane, enzyme, or genetic abnormalities. ${ }^{26-28}$ GCGA induced endocytosis of 5-FU by interacting with the ASGP-R and GA receptors on hepatoma cells surface, and the GCGA nanoparticles had significantly higher anti-drug resistance potential due to a different resistance mechanism from the mechanism against free 5-FU.

In order to evaluate the tumor suppressing potential of GCGA/5-FU nanoparticles in vivo, we treated orthotopic liver transplantation mouse models with GCGA/5-FU for 5 days, and tumor weight was measured. Figure 4A shows that mice injected with GCGA/5-FU had the lowest tumor weight, which was significantly lower than in mice injected with mono-targeting GA-CTS/5-FU and nonliver-targeting CTS/5-FU nanoparticles. This indicated that when compared with the GA-CTS/5-FU and CTS/5-FU nanoparticles, the GCGA nanoparticles induced a more important endocytosis of 5-FU, resulting in higher 5-FU concentration and a lower tumor weight in GCGA/5-FU injected mice. In terms of toxicity, 5-FU caused liver damage and blood cell suppression, while GCGA nanoparticles attenuated these side effects to some extent. Survival analysis suggested that mice injected with GCGA/5-FU nanoparticles had the longest survival time (45 days), while mice injected with GA-CTS/5-FU and CTS/5-FU nanoparticles had a survival time of 37 days and 30 days, respectively, and mice injected with 5-FU had the shortest (24 day) survival time. Results indicated that dual-targeting nanoparticles could mediate more stable endocytosis of 5-FU compared with mono-targeting nanoparticles; and nontargeting nanomaterial showed higher tumor inhibiting capacity than free 5-FU because of its slow release properties.

In conclusion, we synthesized successfully the liver/ tumor-targeting GCGA/5-FU nanoparticles, which slowly released their 5-FU. The in vitro inhibitory effects of GCGA/5-FU nanoparticles on liver tumor were time and dose dependent. GCGA/5-FU nanoparticles significantly reduced the resistance of hepatoma cells to 5-FU, significantly suppressed tumor growth in a liver transplantation mouse model, and prolonged mouse survival time.

\section{Acknowledgments}

This work was supported by Natural Science Foundation of Shanghai (12 nm0502202 and 114119a4700); Pudong New Area Science and Technology Development Fund (PKJ2012Y24); and Minhang District Natural Science Foundation of Shanghai (2010MHZ023).

\section{Disclosure}

The authors report no conflicts of interest in this work.

\section{References}

1. Parkin DM, Bray F, Ferlay J, Pisani P. Estimating the world cancer burden: Globocan 2000. Int J Cancer. 2001;94(2):153-156.

2. Chen WQ, Zheng RS, Zhang SW. Liver cancer incidence and mortality in China, 2009. Chin J Cancer. 2013;32(4):162-169.

3. Siegel R, Naishadham D, Jemal A. Cancer statistics for Hispanics/ Latinos, 2012. CA Cancer J Clin. 2012;62(5):283-298.

4. Sun X, Wu F, Lu W, Zhang ZR. Sustained-release hydroxycamptothecin polybutylcyanoacrylate nanoparticles as a liver targeting drug delivery system. Pharmazie. 2004;59(10):791-794.

5. Zhang X, Zhang X, Yu P, Han Y, Li Y, Li C. Hydrotropic polymeric mixed micelles based on functional hyperbranched polyglycerol copolymers as hepatoma-targeting drug delivery system. J Pharm Sci. 2013;102(1):145-153. 
6. Wang Q, Zhang L, Hu W, et al. Norcantharidin-associated galactosylated chitosan nanoparticles for hepatocyte-targeted delivery. Nanomedicine. 2010;6(2):371-381.

7. Yang W, Mou T, Guo W, et al. Fluorine-18 labeled galactosylated chitosan for asialoglycoprotein-receptor-mediated hepatocyte imaging. Bioorg Med Chem Lett. 2010;20(16):4840-4844.

8. Feng ZQ, Chu X, Huang NP, et al. The effect of nanofibrous galactosylated chitosan scaffolds on the formation of rat primary hepatocyte aggregates and the maintenance of liver function. Biomaterials. 2009;30(14):2753-2763.

9. He ZY, Zheng X, Wu XH, et al. Development of glycyrrhetinic acidmodified stealth cationic liposomes for gene delivery. Int J Pharm. 2010;397(1-2):147-154.

10. Huang W, Wang W, Wang P, et al. Glycyrrhetinic acid-modified poly(ethylene glycol)-b-poly(gamma-benzyl l-glutamate) micelles for liver targeting therapy. Acta Biomater. 2010;6(10):3927-3935.

11. Tian Q, Zhang CN, Wang XH, et al. Glycyrrhetinic acid-modified chitosan/poly(ethylene glycol) nanoparticles for liver-targeted delivery. Biomaterials. 2010;31(17):4748-4756.

12. Kakimoto S, Moriyama T, Tanabe T, Shinkai S, Nagasaki T. Dualligand effect of transferrin and transforming growth factor alpha on polyethyleneimine-mediated gene delivery. J Control Release. 2007;120(3):242-249.

13. Chen H, Li M, Wan T, et al. Design and synthesis of dual-ligand modified chitosan as a liver targeting vector. J Mater Sci Mater Med. 2012;23(2):431-441.

14. Toriumi F, Kubota T, Saikawa Y, et al. Thymidylate synthetase (TS) genotype and TS/dihydropyrimidine dehydrogenase mRNA level as an indicator in determining chemosensitivity to 5-fluorouracil in advanced gastric carcinoma. Anticancer Res. 2004;24(4):2455-2463.

15. Johnson KR, Wang L, Miller MC, Willingham MC, Fan W. 5-Fluorouracil interferes with paclitaxel cytotoxicity against human solid tumor cells. Clin Cancer Res. 1997;3(10):1739-1745.

16. Fang JY, Liu PF, Huang CM. Decreasing systemic toxicity via transdermal delivery of anticancer drugs. Curr Drug Metab. 2008;9(7):592-597.

17. Chen Y, Wang X, Yan Z, Wang J, Luo J, Liu Q. Hepatic arterial infusion with irinotecan, oxaliplatin, and floxuridine plus systemic chemotherapy as first-line treatment of unresectable liver metastases from colorectal cancer. Onkologie. 2012;35(9):480-484.
18. Takahashi Y, Yamashita K, Endo Y, Sasaki T, Mai M. Oral administration of uracil-tegafur (UFT) inhibits liver micrometastasis of human colon cancer in an orthotopic nude mouse model and its early detection system. Surg Today. 2004;34(10):855-859.

19. Oh IH, Min HS, Li L, et al. Cancer cell-specific photoactivity of pheophorbide a-glycol chitosan nanoparticles for photodynamic therapy in tumor-bearing mice. Biomaterials. 2013;34(27):6454-6463.

20. Cheng M, He B, Wan T, et al. 5-Fluorouracil nanoparticles inhibit hepatocellular carcinoma via activation of the p53 pathway in the orthotopic transplant mouse model. PLoS One. 2012;7(10):e47115.

21. Giannì M, Koken MH, Chelbi-Alix MK, et al. Combined arsenic and retinoic acid treatment enhances differentiation and apoptosis in arsenicresistant NB4 cells. Blood. 1998;91(11):4300-4310.

22. Marcucci F, Lefoulon F. Active targeting with particulate drug carriers in tumor therapy: fundamentals and recent progress. Drug Discov Today. 2004;9(5):219-228.

23. Hobbs SK, Monsky WL, Yuan F, et al. Regulation of transport pathways in tumor vessels: role of tumor type and microenvironment. Proc Natl Acad Sci US A. 1998;95(8):4607-4612.

24. Ballet F. Hepatic circulation: potential for therapeutic intervention. Pharmacol Ther. 1990;47(2):281-328.

25. Snow K, Judd W. Characterisation of adriamycin- and amsacrineresistant human leukaemic T cell lines. Br J Cancer. 1991;63(1): $17-28$.

26. Kuo MT, Bao JJ, Curley SA, Ikeguchi M, Johnston DA, Ishikawa T. Frequent coordinated overexpression of the MRP/GS-X pump and gamma-glutamylcysteine synthetase genes in human colorectal cancers. Cancer Res. 1996;56(16):3642-3644.

27. Banerjee D, Mayer-Kuckuk P, Capiaux G, Budak-Alpdogan T, Gorlick R, Bertino JR. Novel aspects of resistance to drugs targeted to dihydrofolate reductase and thymidylate synthase. Biochim Biophys Acta. 2002;1587(2-3):164-173

28. Violette S, Poulain L, Dussaulx E, et al. Resistance of colon cancer cells to long-term 5-fluorouracil exposure is correlated to the relative level of Bcl-2 and Bcl-X(L) in addition to Bax and p53 status. Int J Cancer. 2002;98(4):498-504.
International Journal of Nanomedicine

\section{Publish your work in this journal}

The International Journal of Nanomedicine is an international, peerreviewed journal focusing on the application of nanotechnology in diagnostics, therapeutics, and drug delivery systems throughout the biomedical field. This journal is indexed on PubMed Central, MedLine, CAS, SciSearch $\AA$, Current Contents ${ }^{\circledR} /$ Clinical Medicine,

\section{Dovepress}

Journal Citation Reports/Science Edition, EMBase, Scopus and the Elsevier Bibliographic databases. The manuscript management system is completely online and includes a very quick and fair peer-review system, which is all easy to use. Visit http://www.dovepress.com/ testimonials.php to read real quotes from published authors. 